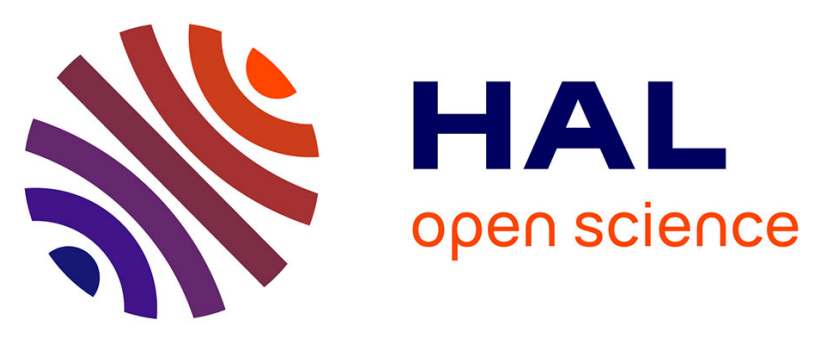

\title{
Determination of the elemental composition of micrometric and submicrometric particles levitating in a low pressure Radio-Frequency plasma discharge using Laser-Induced Breakdown Spectroscopy
}

Christophe Dutouquet, Gaëtan Wattieaux, L. Meyer, Emeric Frejafon, L. Boufendi

\section{To cite this version:}

Christophe Dutouquet, Gaëtan Wattieaux, L. Meyer, Emeric Frejafon, L. Boufendi. Determination of the elemental composition of micrometric and submicrometric particles levitating in a low pressure Radio-Frequency plasma discharge using Laser-Induced Breakdown Spectroscopy. Spectrochimica Acta Part B: Atomic Spectroscopy, 2013, 83-84, pp.14-20. 10.1016/j.sab.2013.01.007 . ineris00961798

HAL Id: ineris-00961798

https://hal-ineris.archives-ouvertes.fr/ineris-00961798

Submitted on 4 Apr 2014

HAL is a multi-disciplinary open access archive for the deposit and dissemination of scientific research documents, whether they are published or not. The documents may come from teaching and research institutions in France or abroad, or from public or private research centers.
L'archive ouverte pluridisciplinaire HAL, est destinée au dépôt et à la diffusion de documents scientifiques de niveau recherche, publiés ou non, émanant des établissements d'enseignement et de recherche français ou étrangers, des laboratoires publics ou privés. 
Determination of the elemental composition of micrometric and submicrometric particles levitating in a low pressure RF (Radio-Frequency) plasma discharge using LIBS (Laser-Induced Breakdown Spectroscopy)

Authors: C. Dutouquet ${ }^{\mathrm{a}, *}$, G. Wattieaux ${ }^{\mathrm{b}}$, L. Meyer ${ }^{\mathrm{b}}$, E. Frejafon ${ }^{\mathrm{a}}$ and L. Boufendi ${ }^{\mathrm{b}}$

a Institut National de l'Environnement Industriel et des Risques (INERIS/DRC/CARA/NOVA), Parc Technologique Alata, BP 2, 60550 Verneuil-En-Halatte, France

${ }^{\mathrm{b}}$ Groupe de Recherches sur l'Énergétique des Milieux Ionisés (GREMI) UMR 6606, CNRS/Université d’Orléans, 14 rue d'Issoudun, BP 6744, 45067 Orléans Cedex 2, France

*Corresponding author: Christophe Dutouquet

Christophe.dutouquet@ineris.fr

Tel: +33344618146

Fax: +33 344556872 


\begin{abstract}
The LIBS (Laser-Induced Breakdown Spectroscopy) technique has shown its potential in many fields of applications including that of aerosol analysis. The latter is usually carried out on the particle flow, thereby allowing quantitative detection in various experimental conditions such as ambient air analysis or exhaust stack monitoring, to name but a few. A possible alternative method for particle analysis has been experimented combining a low pressure RF (Radio Frequency) plasma discharge with the LIBS technique. Such approach has two peculiar features in comparison to usual LIBS analysis. First, the particles injected in the RF plasma discharge are trapped in levitation. Second, the analysis is performed at a reduced pressure of around $1 \mathrm{mBar}$. LIBS detection at such low pressure has this peculiar that particle vaporization is assumed to be achieved through direct laser particle interaction whereas it is caused by a laser-induced plasma ignited in the gas at atmospheric pressure. The use of such particle trap could allow improving particle sampling, making organic particle analysis possible (by using an inert gas for RF plasma ignition) and even (depending on the pressure) obtaining a better signal to noise ratio. Detection of the elements nanoparticle agglomerates were made of following their injection in the RF discharge has demonstrated the feasibility of such approach. Future experiments are intended to explore its potentialities when tackling issues such as process control or ambient air monitoring.
\end{abstract}

Keywords: agglomerates of composite nanoparticles, RF discharge cell, LIBS (Laser-Induced Breakdown Spectroscopy), reduced pressure, particles in levitation, particle trap 


\section{Introduction}

Nanotechnology is a dynamic, growing field of activity in many industrialized countries. Considerable investments have been made in research and development in this fast-developing industry said to be that of the $21^{\text {st }}$ century. Expectations are high to see rising a new generation of products with remarkable features and numerous new applications. Many economical sectors are to be impacted, of which areas related to materials, pharmaceutical and electronics to name but those the most frequently cited. Everyday life may be affected in many ways as well $[1,2]$.

Elaborating nanostructured materials with advanced properties, calls for nanoparticle production processes. Particles were indeed demonstrated to gain specific properties when designed at nanometric scale displaying for instance unusual strength, conductivities or optical properties. These are usually incorporated, mixed with other compound so that the resulting material gain enhanced functionalities. To take but a few examples, engineered nanoparticles of titanium dioxide are used for self-cleaning windows. Nanoparticles of silver are embedded in medical dressings whereas those of $\mathrm{SiC}$ are employed to design high temperature resistant materials in the nuclear industry.

Along with the rise of nanoparticle production grows the need for nanometrology. Control of nanoparticle properties and functionalities entails the development of adapted new instruments. Furthermore, though research remains active to keep production processes reliable and to assure constant quality production, disruptions that may occur while synthesizing ENPs (Engineered nanoparticles) and affect the resulting lots are not to be underestimated. Possible losses of ENP batches caused by sudden diversion from the usual operating conditions resulting in the ENPs not displaying expected properties and therefore useless for targeted applications clearly highlight the need for on-line monitoring instruments.

There exist various manufacturing processes leading to nanoparticle production. Among these, processes allowing continuous synthesis such as laser pyrolysis arouse interest for production at industrial scale. Details on this technique are not described here for they were presented in a previous paper [3]. This method is deemed very promising for nanocomposite particle synthesis owing to its particular qualities. Properties of these nanoparticles strongly depend on the relative abundance of each element (or stoichiometry) they are made of. Though laser pyrolysis is known to assure a good control of compound stoichiometry, diversion from the expected chemical composition is not to be excluded, as emphasized above. On-line Monitoring of composite nanoparticle synthesizing seems therefore necessary. 
The LIBS technique is considered as a potential candidate to deal with such issues $[4,5,6,7]$. LIBS consists in focusing a powerful laser pulse on a material (solid, liquid, gas, nanoparticle flow) whose elemental composition is to be determined. The strong heating of the sample at the focusing spot leads to the ignition of a plasma. Simultaneous detection of all the elements the analyzed material is made of is then achieved through optical emission spectroscopy. Being all optical, LIBS is not intrusive and remote or stand-off analyses are even possible. Besides, samples do almost not need preparation. The aforementioned features do make LIBS a promising analytical chemistry method intended to be operated at industrial sites. This issue of composite particle stoichiometry monitoring was first dealt with using Laser-Induced Breakdown Spectroscopy (LIBS) right on the nanoparticle flow [3]. With the final aim in view to enhance detection and to facilitate possible other laser diagnostics, preliminary experiments were carried out coupling the LIBS analyzer with a low pressure RF (Radio Frequency) plasma discharge acting as a particle trap. Agglomerates of composite nanoparticles of SiC and $\mathrm{Al}_{2} \mathrm{O}_{3}$ were injected into the $\mathrm{RF}$ plasma discharge where they were trapped in levitation $[8,9,10,11]$. LIBS detection was eventually achieved with a satisfying signal to noise ratio at a reduced pressure of $0.25 \mathrm{mBar}$ instead of the atmospheric pressure. The observation of the particle cloud was this peculiar that it did not reveal a bright flash of light, consequence of the ignition of a laser-induced plasma in a gas at a pressure of around $1 \mathrm{~atm}$. Accordingly, particle vaporization was concluded to be caused by direct interaction between laser pulses and the particles whereas it is achieved by the laser-induced plasma when operating at atmospheric pressure. Thus, this coupling presents several advantages. All injected particles are trapped and can potentially be analyzed, thereby improving sampling. Organic particle analysis is made possible without interferences with the $\mathrm{C}, \mathrm{H}, \mathrm{N}, \mathrm{O}$, elements already present in the air when using an inert gas such as argon or helium for RF plasma generation. In addition, the signal-to-noise ratio may be better at reduced pressure than at atmospheric pressure. Last but not least, other laser diagnostics may be envisaged as well.

In this paper, we report the preliminary results obtained when operating such coupling. A first appraisal of the LIBS signal evolution as a function of time delay and pressure was made. Rough estimations of particle number concentrations and plasma temperature were made as well. Eventually, the obtained results demonstrate the feasibility of chemical identification of elements constituting particles combining LIBS and an RF plasma. Experiments specifically dedicated to composite nanoparticle stoichiometry determination remain to be done. Such set up could be envisaged to be integrated in a pyrolysis production unit. Laser particulate interaction experiments using an RF discharge have already been investigated in past studies $[12,13]$. However, chemical identification of particles is to our knowledge reported for the first time in such experimental conditions. 


\section{Experimental Set-up}

The experimental set-up is presented on figure 1 .

\subsection{The RF discharge cell}

The RF cell presents itself as a $10 \mathrm{~cm}$ wide and $10 \mathrm{~cm}$ high rectangular-shaped stainless steel cell fitted with four optical windows allowing particle observation using optical diagnostics. A pair of 6 centimeter diameter parallel disc-shaped electrodes with a $5 \mathrm{~cm}$ gap separation is enclosed within the cell in horizontal position. The electrode from the bottom is connected to the ground, while that of the top is fed by an RF generator. The latter operates at $13.56 \mathrm{MHz}$ delivering a power of $4 \mathrm{~W}$ through a matching network assuring impedance adaptation between the generator and the plasma. The RF cell is vacuumed using a BOC Edwards XDS-5 pump down to a pressure of $0.25 \mathrm{mBar}$. Background gas is then flowed in from the top of the cell using a precision valve. Air was favored in these experiments instead of an inert, plasmagene gas such as argon for a micro leak was detected when starting the experiments. A Baratron gauge (MKS Instruments) monitors the pressure within the chamber. A particle injector positioned on the top of the RF cell and described below was employed to flow in particles into the low pressure plasma. The RF cell rested upon locknuts screwed on four M6 threaded posts fixed on the experimental bench. Laser probing along a vertical axis was therefore achieved by positioning the locknuts and therefore the RF cell they support at the desired vertical position.

\subsection{The nanoparticle injector}

The injection unit is made up of two components, namely the injector mechanism and a small reservoir containing agglomerated ENP powders. The $1 \mathrm{~cm}$ diameter and $0.5 \mathrm{~cm}$ height cylindrical-shaped metallic receptacle was manually filled in, taking precautions inherent to ENP handling. Manipulations were conducted in a hood wearing gloves. The receptacle is capped with a $5 \mu \mathrm{m}$ step mesh grid facing the inner plasma RF discharge chamber when placed in operational position. It was made integral with a metallic rod, part of the injection mechanism. When activated, an electro magnet makes the rod and therefore the receptacle move up and down along its axis, thereby exerting a shaking action on the powders, forcing them through the grid right into the plasma discharge. One millimeter metal beads laid within the reservoir helped particles passing through the grid. 


\subsection{Laser spectroscopy set-up}

Laser pulses with energies of $300 \mathrm{~mJ}$ and durations of $6 \mathrm{~ns}$ fired by a $1064 \mathrm{~nm}$ Q-switched laser (Quantel Brilliant) operating at $20 \mathrm{hz}$ were first spatially expanded to three times the initial beam diameter. Such increase in radius aims at reducing the power density deposited on the window when focusing laser pulses inside the cell. A dichroïc mirror fully reflective at $1064 \mathrm{~nm}$ and $355 \mathrm{~nm}$ but otherwise transparent directed the beam toward a $85 \mathrm{~mm}$ focal length lens allowing focusing laser pulses within the cell. The mirror was positioned so as to make a 90 degree angle between the optical axis of the beam expander and that of the focusing lens. Such optical path makes light collection along the focusing axis possible, given that the dichroïc mirror is transparent to almost all wavelengths but that of the laser. Light emitted by particle disintegration was then collected through the very lens utilized for laser focusing and focused on the entrance of a $600 \mu \mathrm{m}$ core diameter optical fiber (Ocean Optics) using another $50 \mathrm{~mm}$ focal length lens. Optical emission spectra were retrieved from a spectrometer (iHR320) equipped with a gated intensified CCD camera (Andor iStar model DH734-18F-03). Time resolved measurements are made possible with such detector by triggering signal recording when the laser pulses are fired and recorded over the required duration by selecting the appropriate integration time (with accessible time resolution of around $2 \mathrm{~ns})$.

\section{Foreword}

\subsection{Brief description of particle batches}

The experiments were performed on two different batches of composite nanoparticles. These first tests were run with $\mathrm{SiC}$ and $\mathrm{Al}_{2} \mathrm{O}_{3}$ powders. Both nanoparticle lots were synthesized by laser pyrolysis technique [3]. Particle characteristics were observed using SEM (Scanning Electron Microscopy) prior to these experiments. SEM observations showed that SiC agglomerates were formed. Their median and upper size values were found to be of around 800 and $1200 \mathrm{~nm}$ respectively. Particles of $\mathrm{Al}_{2} \mathrm{O}_{3}$ displayed a rather monodisperse distribution with sizes of around $350 \mathrm{~nm}$. Their morphologies proved to be different as particles of $\mathrm{Al}_{2} \mathrm{O}_{3}$ are rather spherical whereas those of $\mathrm{SiC}$ present a more complex structure.

\subsection{Injection procedure}

Nanoparticle injection operation is carried out while the RF discharge is being operated. A white light lamp illuminating one of the four windows and making the hovering nanoparticle cloud visible through diffusion was employed as a basic monitoring tool (based on eye observation). Through former studies, particles are 
known to gain a negative charge when injected in an RF plasma cell $[14,15]$. This has two major consequences on their behavior. First, agglomerates tend to de-agglomerate under electrostatic repulsion forces. Second, particles were demonstrated to levitate within the RF plasma discharge $[8,9,10,11]$. The two main forces exerted on particles, that of gravity and the electrostatic force originating from the electric field created by the RF discharge, happen to balance [16], thereby resulting in particles being in levitation. Trapping occurs at a height mainly dependent on particle surface [17]. As a consequence, particles distribute according to their sizes along the cylindrical symmetry axis passing through the electrodes, larger particles being preferentially located near the bottom electrode. Conversely, smaller particles tend to occupy the upper part of the cloud hovering in the RF cell.

\section{Results and discussion}

\subsection{Temporal settings and spectrum acquisition mode}

The LIBS signal was recorded after each laser shot by selecting proper time delay and integration time values. The latter two parameters were arbitrarily set to $1 \mu$ s and $5 \mu$ s respectively when running the first tests for time delays optimizing the LIBS signal were not known yet. Detector settings were arranged so that emission stemming from each laser shot could be recorded in individual spectra. Such recording mode presents the advantage of allowing monitoring of spectroscopic signal emission from individual particle hit by laser pulses.

\subsection{Comments on injection of $\mathrm{Al}_{2} \mathrm{O}_{3}$ and $\mathrm{SiC}$}

Two first series of experiments were successively carried out by probing $\mathrm{Al}_{2} \mathrm{O}_{3}$ and $\mathrm{SiC}$ clouds of particles with the aforementioned settings. A total of five hundred individual spectra corresponding to the same number of laser shots were recorded for each series. A first basic diagnostic was performed looking at the nanoparticle cloud from the beginning to the end of the injection procedure. Direct eye observation by white light diffusion suggested that the $\mathrm{Al}_{2} \mathrm{O}_{3}$ cloud was much denser than that of SiC. Such difference in concentration recurrently observed throughout the whole campaign of experiments may be accounted for as follows. SiC particle injection happened to be arduous. As already underlined above, SEM observations of the suspended particles revealed that $\mathrm{Al}_{2} \mathrm{O}_{3}$ particles are spherical-shaped whereas non-sphericity of SiC particles was blatant. Connecting these two facts hints that non sphericity of SiC particles may preclude them from flowing smoothly through the grid of the reservoir. Spherical-shaped $\mathrm{Al}_{2} \mathrm{O}_{3}$ particles are contrariwise more prone to traverse the holey grid. The $\mathrm{Al}_{2} \mathrm{O}_{3}$ ENP cloud was therefore always denser than that of $\mathrm{SiC}$. 
The particle cloud was also observed when probed by the laser pulses. This observation did not reveal bright light emission such as that usually seen when igniting a laser-induced plasma at atmospheric pressure. It strongly suggested that particle vaporization is the consequence of direct laser particle interaction within the focusing volume whereas it is mainly caused by the laser-induced plasma at atmospheric pressure. This point is worth emphasizing for LIBS literature related to particle analysis is mainly performed at atmospheric pressure. LIBS signal is assumed originating from a microplasma formed when particles are vaporized.

\subsection{Analysis of recorded spectra}

Following injection, both clouds were interrogated by LIBS. The results are presented on figure 2 . Al lines at 394.40 and $396.15 \mathrm{~nm}$ (see table 1) were recorded while interrogating the $\mathrm{Al}_{2} \mathrm{O}_{3}$ ENP cloud (figure 2a). Though oxygen lines could be detected using optical emission spectroscopy, these were not selected for a first trial. The impossibility to tell apart oxygen of the air used as background gas in the RF discharge from that contained in the alumina ENPs resulted in our not insisting on trying to detect these lines. Data analysis demonstrated that each laser shot caused Al lines to rise from the background noise in each corresponding spectra. This result was achieved regardless of the vertical position of the focal volume above the bottom electrode. Detection of the elements constituting $\mathrm{SiC}$ (figure 2b) turned out to be different in every way. Attention was focused on the detection of $\mathrm{Si}$ lines and that at $288.15 \mathrm{~nm}$ (table 1) emerged when scanning vertically the ENP cloud along the RF cell symmetry axis. Carbon detection was not achieved in the course of our experiments. Difficulties inherent to carbon line detection are discussed further below. Eventually, recording of the Si line could only be fulfilled when probing the bottom of the cloud. Contrary to the observations made when dealing with the $\mathrm{Al}_{2} \mathrm{O}_{3}$ particle cloud, successive recordings of single spectra corresponding to unique shots did not always occasion line emergence. Only a slight fraction of the total number of spectra exhibited the searched line.

The obtained results point out a predictable influence of 1) the position of the focal volume along the symmetry axis of the RF discharge and 2) the concentration within the cloud on particle detection.

The detection dependence with the position of the focal volume seems consistent with the aforementioned described pattern of size distribution of particles along the symmetry axis of the RF discharge, larger particulates of SiC being located at the bottom of the cloud. Larger particles are very likely to yield larger amount of mass when vaporized by a laser pulse. As spectroscopic detection sensitivity depends on this mass yield, particles with larger sizes are more likely to be detected than those of tinier radius. 
Probability of detection is therefore expected to decrease as the focused volume or sampling zone moves upward from the bottom plate electrode. Given these observations, a rough estimation of the sizes of the detected particles can be made. Sizes of the largest particles were found to be of around $1.2 \mu$ m under SEM analysis. As previously explained, particles immersed within the RF plasma are known to repel one another and therefore do not agglomerate to form larger particles. As a consequence, sizes of particles leading to signal emission are estimated of around $1 \mu \mathrm{m}$ or so. Such dependence was not observed when dealing with the $\mathrm{Al}_{2} \mathrm{O}_{3}$ cloud. Though $\mathrm{Al}_{2} \mathrm{O}_{3}$ particle distribution was rather monodisperse, particles were not all found to be located at the same height in the RF discharge as expected. The rather high concentration of particles squeezed into the cell (the injection being facilitated by particle shape as previously explained), thereby leading to the formation of a large, dense cloud may explain their not distributing along the symmetry axis according to the pattern briefly described in section 3.2.

$\mathrm{SiC}$ probing presents another peculiarity in comparison with that of $\mathrm{Al}_{2} \mathrm{O}_{3}$. Data processing related to the latter showed that all laser shots did occasion aluminum line detection. Conversely, careful examination of the $\mathrm{SiC}$ spectra makes stand out the non systematic rise of Si line for each laser shot. Only $9 \%$ of the recorded spectra displayed Si detection. This seems obviously connected to particle number density. Indeed, line emission can only occur when a particle is sampled within the laser focusing volume. The lower the particle number density, the lower the sampling rate and therefore the probability to record lines corresponding to the elements the probed particles are made of. This observation has two consequences. First, it points out that detection of single particle is possible. Indeed, analysis of series of spectra shows that several hundreds of ms can elapse between two consecutive recorded lines. This fuels the hypothesis of single particle detection, the latter being entirely vaporized or repelled out of the focusing area before the next pulse. Signal recording recurs when a particle pops up in the focusing area again. Single particle detection may be of great interest when it comes to dust formation within plasma reactors such as RF discharges. Second, this mode of detection both inherent to the transient nature of the LIBS signal and to the discrete nature of particle implies a specific data processing to make the most of the recorded spectra. Stochiometry determination may motivate the obtaining of a spectroscopic signal with high signal to background ratio. The usual way to deal with such issue consists of ensemble-averaging all recorded individual spectra. Still, such approach may be inadequate with low sampling rates, as already emphasized by Hahn et al. [18] when carrying out LIBS experiments at atmospheric pressure. When faced with low sampling rates, discarding spectra free of signal prior to averaging is beneficial to the intensity of signal to background ratio. Applying such procedure reported as conditional analysis [18] is 
undoubtedly helpful when less than $20 \%$ of individual spectra contain signal. Average spectra of Al and Si lines were therefore obtained applying ensemble-averaging and conditional analysis [19] respectively. They are presented on figures $3 \mathrm{a} \& 3 \mathrm{~b}$ and display satisfying signal to background ratio.

\subsection{Particle concentration estimation}

Though particle number concentrations cannot easily be determined in the framework of these experiments, their limit values (upper limit for $\mathrm{SiC}$ and lower limit for $\mathrm{Al}_{2} \mathrm{O}_{3}$ ) were roughly estimated combining the stochastic nature of particle sampling and a calculation of the focusing volume. The latter is approximately expressed using the following equation [20], assuming a Gaussian radial profile:

$$
V=19.2(f / D)^{4} \lambda^{3}
$$

where $f$ is the focusing lens length, $D$ the laser beam diameter, and $\lambda$ the laser beam wavelength. Taking into account that a lens with focusing length of $85 \mathrm{~mm}$ was used and that the laser beam diameter and laser wavelength values were of around $18 \mathrm{~mm}$ and $1064 \mathrm{~nm}$ respectively, the focal volume was estimated to be of around $10^{-8} \mathrm{~cm}^{-3}$. Eventually, this estimation of the focal volume combined with statistical considerations on line recording allows assessing number densities within the RF discharge. Indeed, systematic particle hit was observed through $\mathrm{Al}_{2} \mathrm{O}_{3}$ ENP spectroscopic measurement leading to a sampling rate of $100 \%$ whereas the latter value fell to $9 \%$ when probing the less concentrated cloud of SiC. The former case highlights the fact that particles were always present within the focusing volume when irradiating $\mathrm{Al}_{2} \mathrm{O}_{3}$ whereas this condition was not fulfilled when letting laser pulses passing through the $\mathrm{SiC}$ cloud. Considering that the average number of particles within the focusing volume may be calculated using the following formula:

$$
N=c V
$$

where $c$ is particle number concentration and $V$ the focusing volume, an estimation of limit values for each case may be given. Assuming that there was at least always one $\mathrm{Al}_{2} \mathrm{O}_{3}$ particle within the focusing volume, the corresponding minimum particle number concentration may be assessed with a value of around $10{ }^{8}$ part per cc or more. The SiC concentration is contrariwise estimated to be lower than the latter value given the sampling rate obtained when probing $\mathrm{SiC}$ particles.

\subsection{LIBS signal evolution as a function of time delay}

Temporal evolution of aluminum lines was investigated when analyzing $\mathrm{Al}_{2} \mathrm{O}_{3}$ particles. The results are presented on figure 4 where the obtained spectra were ensemble-averaged over one thousand and four hundred 
laser shots. First, these spectra display very low background emission [21] remaining at the same intensity level for time delays ranging from $100 \mathrm{~ns}$ to $5 \mu \mathrm{s}$. In comparison is the background intensity of spectra recorded when detecting particles disintegrated at atmospheric pressure within a hot laser-induced plasma much higher for similar time delays. Second, time delays maximizing the LIBS signal were found to be much shorter than those usually used when carrying out LIBS experiments at atmospheric pressure. Aluminum line intensities were found to increase with decreasing time delays, the maximum LIBS intensity being recorded for the shortest time delay employed in the allotted time of these preliminary experiments, namely $100 \mathrm{~ns}$. Values of a few to a few dozens of microseconds were contrariwise reached at atmospheric pressure. This might be accounted for by the difference in plasma temperature. Indeed, each line corresponding to the elements particles may be made of were shown to maximize their intensity at a preferential temperature $[22,23,24]$. According to the plasma cooling speed, the time necessary for a line of a given element to reach its preferential temperature does impose the time delay for which the LIBS signal is to the maximum. Examination of the spectra of figure 4 reveals an additional line at $391.44 \mathrm{~nm}$ when recording spectra 100 and $500 \mathrm{~ns}$ subsequently to laser pulses. The latter stands for nitrogen molecular ion, whose spectroscopic features are reported in table 2. Line intensities of this molecule appear to taper off as a function of time delay and are therefore concluded to be related to LIBS signal. Such correlation tends to indicate that molecular emission originates from LIBS signal and not from the RF plasma discharge. The atoms of nitrogen dissociated in the early times of particle ablation within the focusing volume recombine when the microplasma cools down, thereby resulting in the formation of the $\mathrm{N}_{2}{ }^{+}$ionized, and excited molecule. Temperature of a plasma may be inferred through such molecular emission [25]. They usually do not exceed a few thousand degree Kelvin, range within which the microplasma temperature may vary. Though neither the exact microplasma temperature value nor the time delay maximizing the LIBS signal were precisely determined, the obtained results seem consistent with those of a previous study at atmospheric pressure showing a preferential temperature of around $7500 \mathrm{~K}$ to optimize the $\mathrm{Al} 396 \mathrm{~nm}$ line recording [22]. As to possible more intense background emission that may be recorded in the early time delay range not explored in the present paper, literature sheds light on possible continuum emission. Stoffels et al. [12] concluded, when investigating laser-particulate emission interactions in a dusty RF plasma, that blackbody-like emission observed when irradiating particles with $\mu$ s and ns laser pulses originated from particle surfaces and by no means by excited atomic vapor expanding fast under reduced pressure. The latter would be worth studying in order to ascertain or not the need for gated detectors when carrying out such experiments. 


\subsection{Discussion on difficulties of carbon detection and carbon line emission as a function of pressure}

As explained above, the final objective of these experiments is to assess stoichiometry, thereby necessitating the detection of all the elements composing the powders. Carbon line at $247.85 \mathrm{~nm}$ was not observed when vaporizing $\mathrm{SiC}$ particles in the $\mathrm{RF}$ cell under pressure of $0.25 \mathrm{mBar}$. Several reasons may have caused this line not to be seen under the aforementioned conditions. Spectroscopic characteristics of the 247.85 $\mathrm{nm}$ carbon line make it harder to detect than aluminum and silicon given its transition probability and the upper state energy level (see table 1). In addition, this line was found to be maximized for a temperature higher than that found for aluminum, namely around $11000 \mathrm{~K}$ [22]. Moreover, carbon happens to recombine with nitrogen in the early times of plasma formation, thereby reducing the number of carbon emitters. These latter two points suggest the selection of a short time delay. In addition to these intrinsic properties of the transitions, the window transmittance of the RF cell was measured to be of around 30\%. Eventually, the ICCD response efficiency decreasing with decreasing wavelength ends up listing the weaknesses hindering carbon detection.

Based on this failure to detect the expected line, decision was made to try again to detect carbon using an alternative experimental set-up. The RF discharge reactor was changed for a quartz four-way cross chamber. The injector was fixed on its top and vacuuming was assured using the very pumping system utilized for all the other experiments. The focused laser pulses were let in through the chamber with the focusing volume positioned right beneath the injector. The injector was activated while the laser was being operated so as falling powders be intercepted by the focusing volume. Spectra were taken following the one shot / one spectrum recording pattern presented above with a 500 ns time delay (the optimized value was not known) and a $7 \mu$ s integration time. Carbon line intensity was monitored as a function of inner chamber pressure. Acquisitions were stopped as the carbon line intensity was fading until signal collection was no longer possible. Some of the resulting spectra are presented on figure 5 for two different pressures. The signal to noise ratio values of the carbon line were found to approximately vary from around 14 to 10 within about two decades of pressure range, namely from 500 to 1 mbar. Such observation suggests that the carbon line could have been detected at $0.25 \mathrm{mBar}$ using proper LIBS settings and set-up. Results obtained in this additional experiment are quite encouraging for the next steps.

\section{Conclusion}

Preliminary experiments aiming at identifying elemental composition of agglomerates of composite ENPs with the final objective to measure their stoichiometry were performed envisaging a new approach. It rests on the combination of a low pressure RF plasma discharge with LIBS. Contrary to the analysis on a particle flow 
at atmospheric pressure, the particles are trapped and maintained in levitation. As the reduced pressure precludes the ignition of a hot plasma in the gas, particle vaporization is therefore assumed to be the consequence of direct laser interaction. Eventually, aluminum and silicon lines recorded in spectra obtained when probing $\mathrm{Al}_{2} \mathrm{O}_{3}$ and SiC ENP clouds were successfully observed. The $\mathrm{Al}_{2} \mathrm{O}_{3}$ particle density within the $\mathrm{RF}$ cell was estimated to be of around $10^{8} \mathrm{~cm}^{-3}$ or more whereas that of $\mathrm{SiC}$ was assessed lower than that. Time resolved recordings performed within the $100 \mathrm{~ns}$ to $5 \mu$ s delay range showed that maximum LIBS intensity was reached for the shortest delay set when carrying out these experiments, namely 100 ns. Time delays maximizing signal to background ratio were concluded to have to be selected close after the end of the laser pulse. Carbon line detection proved to be more difficult. However, its detection is deemed feasible provided proper temporal settings and experimental set-up be employed.

Many questions remain open as to the potentialities of such combination. The dependence of the spectroscopic signal with particle sizes should be investigated. Particle ablation rate could be computed provided the absorption coefficient and enthalpy of evaporation of the targeted compound be found in the literature. Particle size range accessible to such analysis could be envisaged to be lowered through the use of proper laser wavelength and both focusing and collection optical paths. Appraising whether stoichoimetry may be inferred from such analysis will be the next step, involving upgrade of the laser set-up and better understanding of laser particulate matter interaction. Such approach may also be of interest for particle formation monitoring when carrying out experiments in the field of dusty plasmas.

\section{Acknowledgments:}

This research was supported by the French National Research Agency (ANR), the ERDF (European Regional Development Fund) and the French "Région Centre". 


\section{References}

[1] B. Park, Current and future applications of nanotechnology, Issues in environmental Science and Technology 24, The Royal Society of Chemistry (2007)

[2] C. Ostiguy, B. Roberge, C. Woods, B. Soucy, Engineered Nanoparticles, current knowledge about OHS risks and prevention measures, Report R656, IRSST (Institut Robert-Sauvé en Santé et en Sécurité du Travail) (2010)

[3] T. Amodeo, C. Dutouquet, F. Tenegal, B. Guizard, H. Maskrot, O. Le Bihan, E. Frejafon, On-line monitoring of composite nanoparticles synthesized in a pre-industrial laser pyrolysis reactor using Laser-Induced Breakdown Spectroscopy, Spectrochim. Acta Part B 63 (2008) 1183-1190

[4] L.J. Radziemski, T.R. Loree, D. A. Cremers, N.M. Hoffman, Time-resolved laser-induced breakdown spectrometry of aerosols, Anal. Chem. 55 (1983) 1246-1252

[5] D.W. Hahn, M.M. Lunden, Detection and analysis of aerosol particles by laser-induced breakdown spectroscopy, Aerosol Sci. Technol. 33 (2000) 30-48

[6] D. Mukherjee, A. Rai, M. R. Zachariah, Quantitative laser-induced breakdown spectroscopy for aerosols via internal calibration: Application to the oxidative coating of aluminum nanoparticles, J. of Aerosol Sci. 37 (2006) $677-695$

[7] D. W. Hahn and N. Omenetto, Laser-Induced Breakdown Spectroscopy (LIBS), Part I: Review of Basic Diagnostics and Plasma-Particle Interactions:Still-Challenging Issues Within the Analytical Plasma Community, Appl. Spectrosc. 64 (2010) 335-366

[8] H.M. Thomas, G.E. Morfill, V.E. Fortov, A.V. Ivlev, V.I. Molotkov, A.M. Lipaev, T. Hagl, H. Rothermel, S.A. Khrapak, R.K. Suetterlin, M. Rubin-Zuzic, O.F. Petrov, V.I. Tokarev and S.K. Krikalev, Complex plasma laboratory PK-3 Plus on the International Space Station. New J. Phys. 10 (2008) 033036

[9] N. Hayashi, Observation of submicron dust particles trapped in a diffused region of a low pressure radio frequency plasma, Phys. Plasmas 8 (2001) 3051-3058

[10] M. Mikikian, L. Boufendi, A. Bouchoule, H.M. Thomas, G.E. Morfill, A.P. Nefedov, V.E. Fortov and the PKE-Nefedov team, Results in the Laboratory and under Microgravity Conditions, New J. Phys. 519 (2003) 112

[11] G.S. Selwyn, J. E. Heidenreich, and K.L. Haller, Rastered laser light scattering studies during plasma processing: Particle contamination trapping phenomena, J. Vac. Sci. Technol. A 9 (1991) 2817-2824

[12] E. Stoffels, W. W. Stoffels, D. Vender, G. M. W. Kroesen, F. J. De Hoog, Laser-Particulate interactions in a dusty RF plasma, IEEE Transactions on Plasma Sci. 22 (1994) 116-121 
[13] L. Boufendi, J. Hermann, A. Bouchoule, B. Dubreuil, E. Stoffels, W. W. Stoffels, M. L. De Giorgi, Study of initial dust formation in an Ar-SiH4 discharge by laser induced particle explosive evaporation, J. Appl. Phys. 76 (1994) $148-153$

[14] R.L. Merlino, Dusty plasmas and applications in space and industry, Plasma Physics Applied (2006) 73-110

[15] G. Wattieaux, A. Mezeghrane, and L. Boufendi, Electrical time resolved metrology of dust particles growing in low pressure cold plasmas, Phys. Plasmas 18 (2011) 093701

[16] G. Wattieaux and L. Boufendi, Discharge impedance evolution, stray capacitance effect, and correlation with the particles size in a dusty plasma, Phys. Plasmas 19 (2012) 033701, doi: 10.1063/1.3689013

[17] K. D. Bleecker, Modeling of the formation of nanoparticles in dusty plasmas, Ph.D dissertation (2006) Universiteit Antwerpen

[18] D. W. Hahn, W. L. Flower, K. R. Hencken, Discrete particle detection and metal emissions monitoring using laser-induced breakdown spectroscopy, Appl. Spectrosc. 51 (1997) 1836-1844

[19] G. Gallou, J. B. Sirven, C. Dutouquet, O. Le Bihan, and E. Frejafon, Aerosols Analysis by LIBS for Monitoring of Air Pollution by Industrial Sources, Aerosol Sci. Technol. 45 (2011) 908-916

[20] C. G. Parriger, Laser-induced breakdown in gases: experiments and simulation. In: Miziolek AW, Palleschi V, Schechter I (eds) Laser-induced breakdown spectroscopy, fundamentals and applications, Cambridge University Press, Cambridge (2006) pp171-193

[21] A. J. Effenberger, Jr. and Jill R. Scott, Effect of Atmospheric Conditions on LIBS Spectra, Sensors 10 (2010) 4907-4925

[22] T. Amodeo, C. Dutouquet, O. Le-Bihan, M. Attoui, E. Fréjafon, On-line determination of nanometric and sub-micrometric particle physicochemical characteristics using spectral imaging-aided Laser-Induced Breakdown Spectroscopy coupled with a Scanning Mobility Particle Sizer, Spectrochim. Acta Part B 64 (2009) 1141-1152

[23] V. Hohreiter, D.W. Hahn, Calibration effects for laser-induced breakdown spectroscopy of gaseous sample streams: analyte response of gas-phase species versus solid-phase species, Anal. Chem. 77 (2005) 1118-1124

[24] B. T. Fisher, H. A. Johnson, S. G. Buckley, D. W. Hahn, Temporal gating for the optimization of laserinduced breakdown spectroscopy detection and analysis of toxic metals, Appl. Spectrosc. 55 (2001) 1312-1319 [25] A. Chelouah, E. Marode, G. Hartmann, S. Achat, A new method for temperature evaluation in a nitrogen discharge, J. of Phys. D: Appl. Phys. 27 (1994) 940-945

[26] Lines on line. http://physics.nist.gov/PhysRefData/ASD/lines_form.html 
[27] B. Rosen, Données spectroscopiques relatives aux molecules diatomiques (Spectroscopic data related to diatomic molecules) Pergamond, Oxford, 1970 


\section{Figure captions}

Figure 1: Experimental set-up

Figure 2a \& 2b: Examples of spectra (arbitrary selection) acquired consecutive to single laser shots when probing (a) $\mathrm{Al}_{2} \mathrm{O}_{3}$ and (b) $\mathrm{SiC}$ particles levitating in the $\mathrm{RF}$ plasma discharge under $0.25 \mathrm{mBar}$ pressure. The recordings were performed using time delays after the laser pulse and integration time of respectively 1 and $5 \mu \mathrm{s}$ with a total laser shot number of 500. The entrance slit width of the spectrometer was set to $400 \mu \mathrm{m}$ thereby sacrifying spectral resolution to enhance photon collection, the latter being of more importance for these preliminary tests.

Figure 3a \& 3b: Spectra acquired when probing (a) $\mathrm{Al}_{2} \mathrm{O}_{3}$ and (b) $\mathrm{SiC}$ particles hovering in the $\mathrm{RF}$ plasma discharge under $0.25 \mathrm{mBar}$ pressure and ensemble averaged over 500 and 90 (after having used conditional analysis algorithm [19]) laser shots respectively. The recordings were performed using time delays after the laser pulse of $1 \mu \mathrm{s}$ and integration time of $5 \mu \mathrm{s}$. The entrance slit width of the spectrometer was set to $400 \mu \mathrm{m}$, thereby reducing spectral resolution to enhance photon collection, the latter being of more importance for these preliminary tests.

Figure 4: Temporal evolution of ensemble averaged spectra acquired when probing $\mathrm{Al}_{2} \mathrm{O}_{3}$ particles within the $\mathrm{RF}$ plasma discharge under $0.25 \mathrm{mBar}$ pressure. Integration time was fixed to $5 \mu$ s. Spectra were ensemble averaged over 1400 laser shots. The spectrometer entrance slit width was set to $200 \mu \mathrm{m}$ instead of $400 \mu \mathrm{m}$ given the rather fair signal to background ratio obtained when recording these spectra.

Figure 5: Spectra acquired consecutive to one unique laser shot when probing SiC particles at 500 and $1 \mathrm{mBar}$ in the four way cross cell. Time delay and integration time were set to $500 \mathrm{~ns}$ and $7 \mu \mathrm{s}$ respectively. Next to the carbon line can be seen the Si quintuplet lines. The corresponding spectrum is poorly resolved given the wide entrance slit selected to enhance carbon detection. 
Table 1. Wavelengths $\lambda$, transition probabilities $A_{k i}$, lower $E_{i}$ and upper $E_{k}$ energy levels of neutral lines detected when analyzing $\mathrm{SiC}_{\mathrm{x}}$ and $\mathrm{Al}_{2} \mathrm{O}_{3}$ particles (Lines on line [26])

\begin{tabular}{l|l|l|l|l}
\hline Element & $\lambda(\mathrm{nm})$ & $A_{k i}\left(10^{7} \mathrm{~s}^{-1}\right)$ & $E_{i}(\mathrm{eV})$ & $E_{k}(\mathrm{eV})$ \\
\hline Al I & 394.40058 & 4.93 & 0.0 & 3.1427210 \\
& 396.15200 & 9.8 & 0.0138938 & 3.1427210 \\
C I & 247.856 & 2.8 & 2.684011 & 7.684766 \\
Si I & 288.1579 & 21.7 & 0.7809578 & 5.0823456 \\
\hline
\end{tabular}


Table 2. Spectroscopic characteristics of $\mathrm{N}_{2}{ }^{+}$molecular line including bandhead wavelength $\lambda$, upper electronic state $T_{e}$ and transition probability $A_{k i}$. (Rosen, 1970 [27])

\begin{tabular}{l|l|l|l|l|l|l}
\hline Species & System & Transition & Band & $\lambda(\mathrm{nm})$ & $T_{e}(\mathrm{eV})$ & $A_{k i}\left(10^{8} \mathrm{~s}^{-1}\right)$ \\
\hline $\mathrm{N} 2+$ & FNS & $\mathrm{B}^{2} \Sigma_{\mathrm{u}}^{+}-\mathrm{X}^{2} \Sigma_{\mathrm{g}}^{+}$ & $(0,0)$ & 391.44 & 3.17 & -- \\
\hline
\end{tabular}




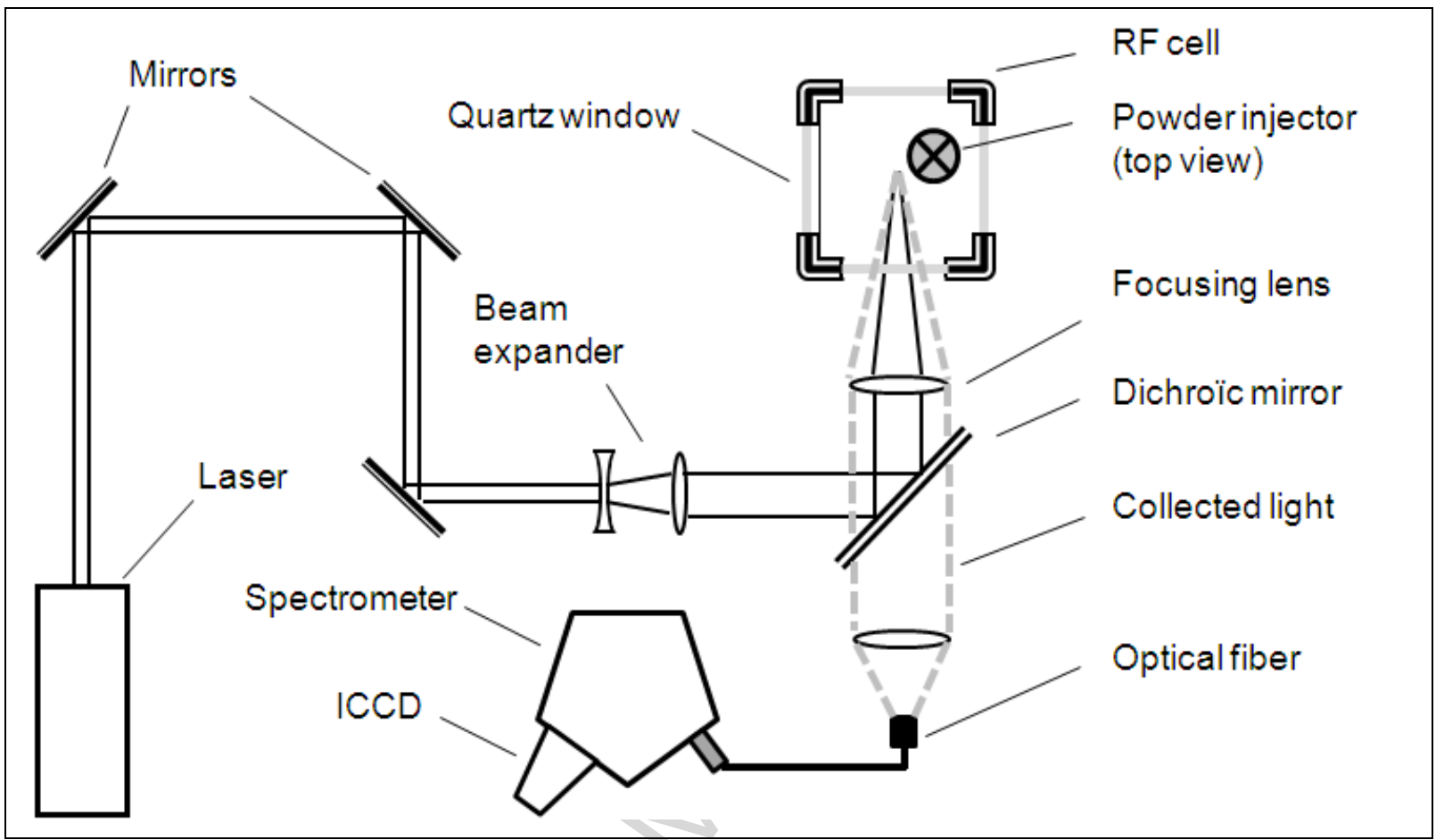

Figure 1 


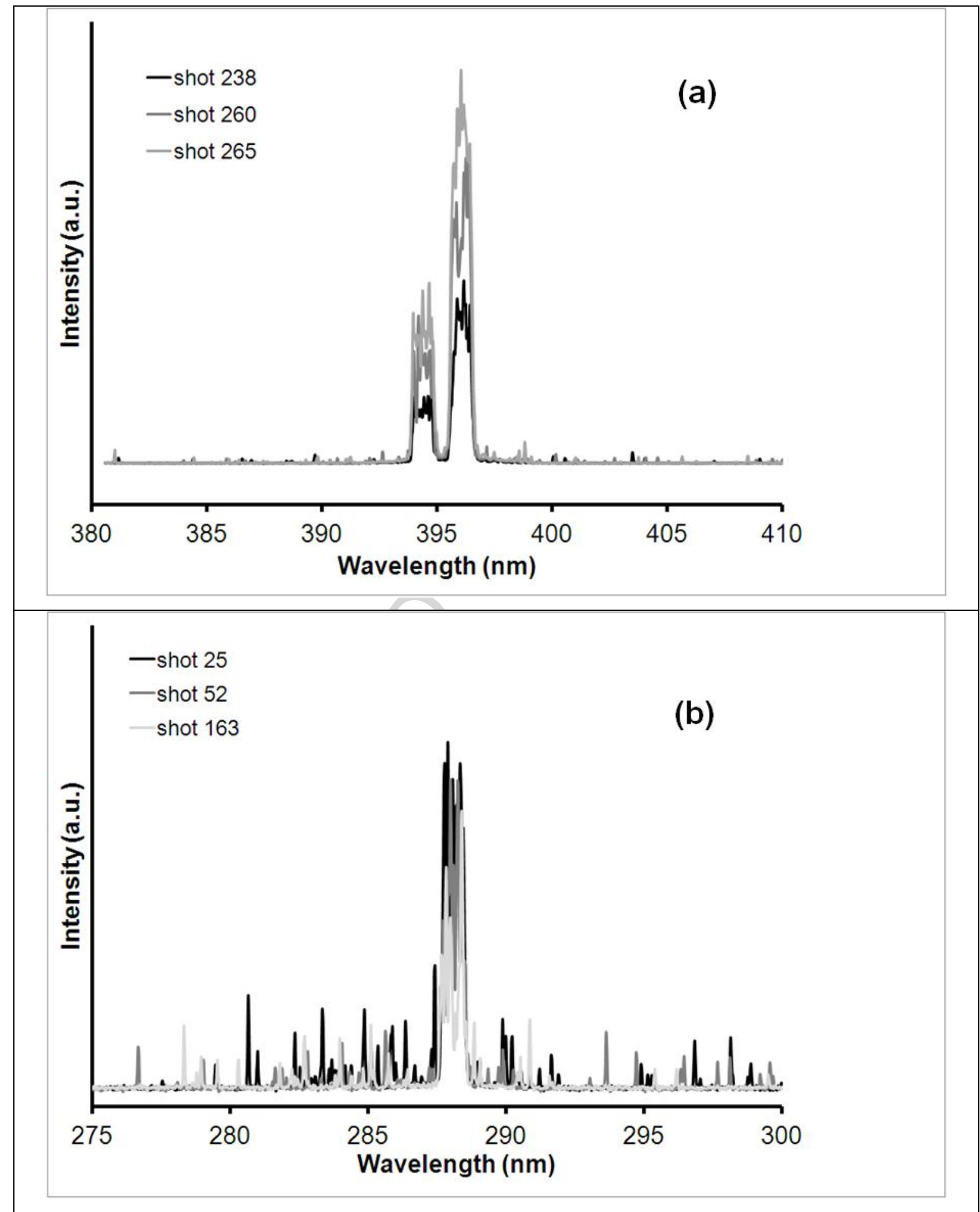

Figure $2 \mathrm{a} \& 2 \mathrm{~b}$ 


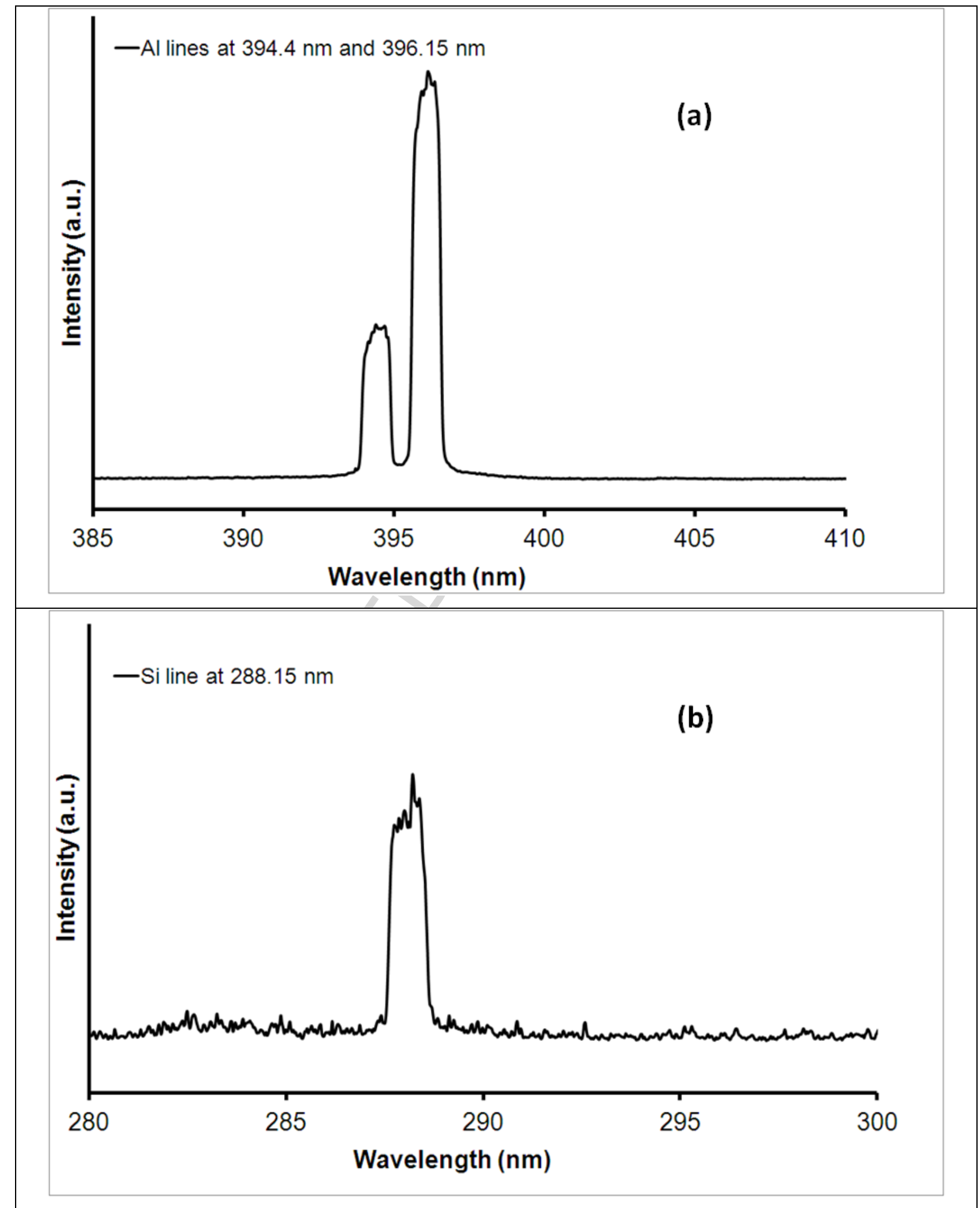

Figure $3 a$ and $3 b$ 


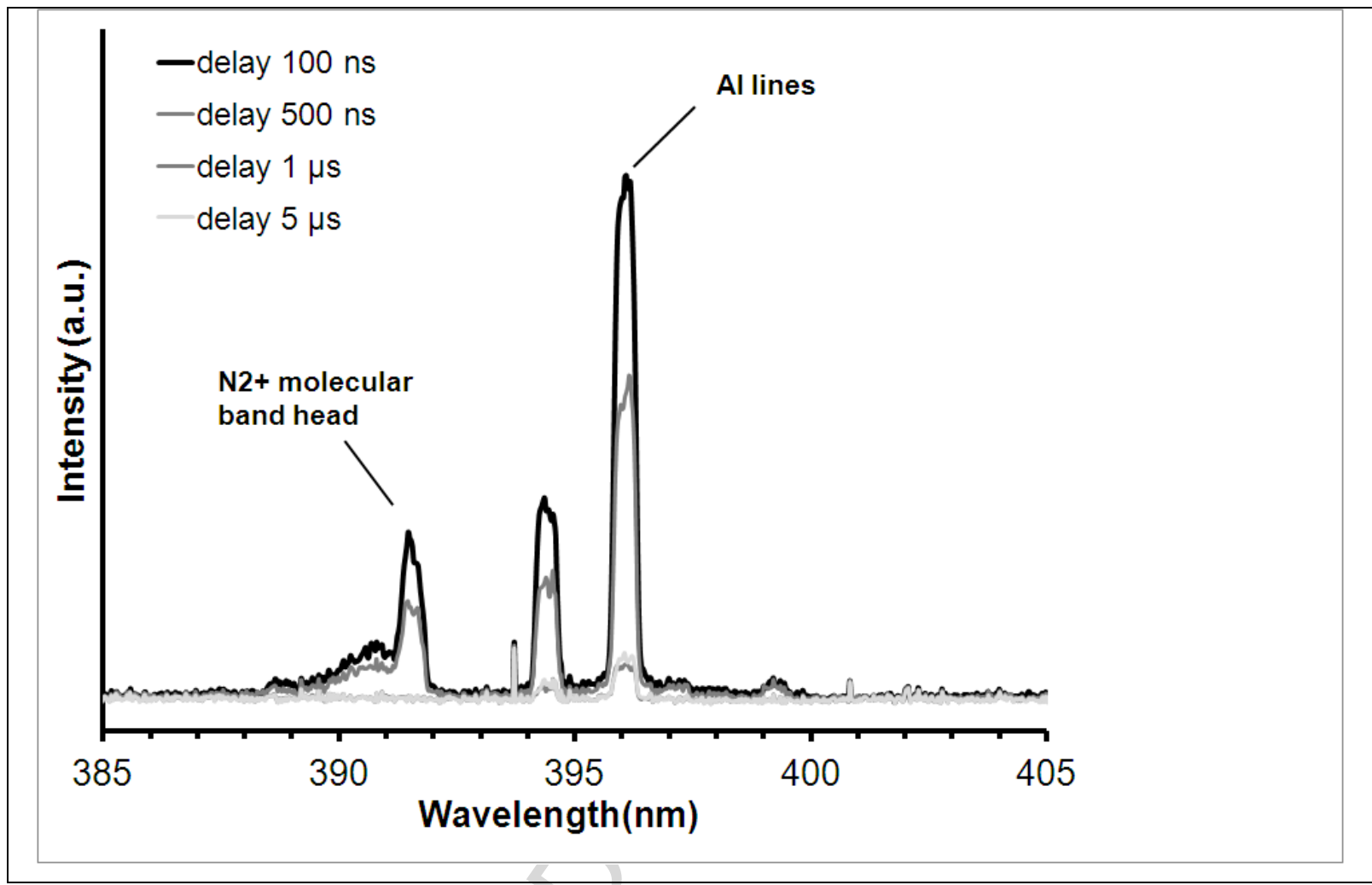

Figure 4

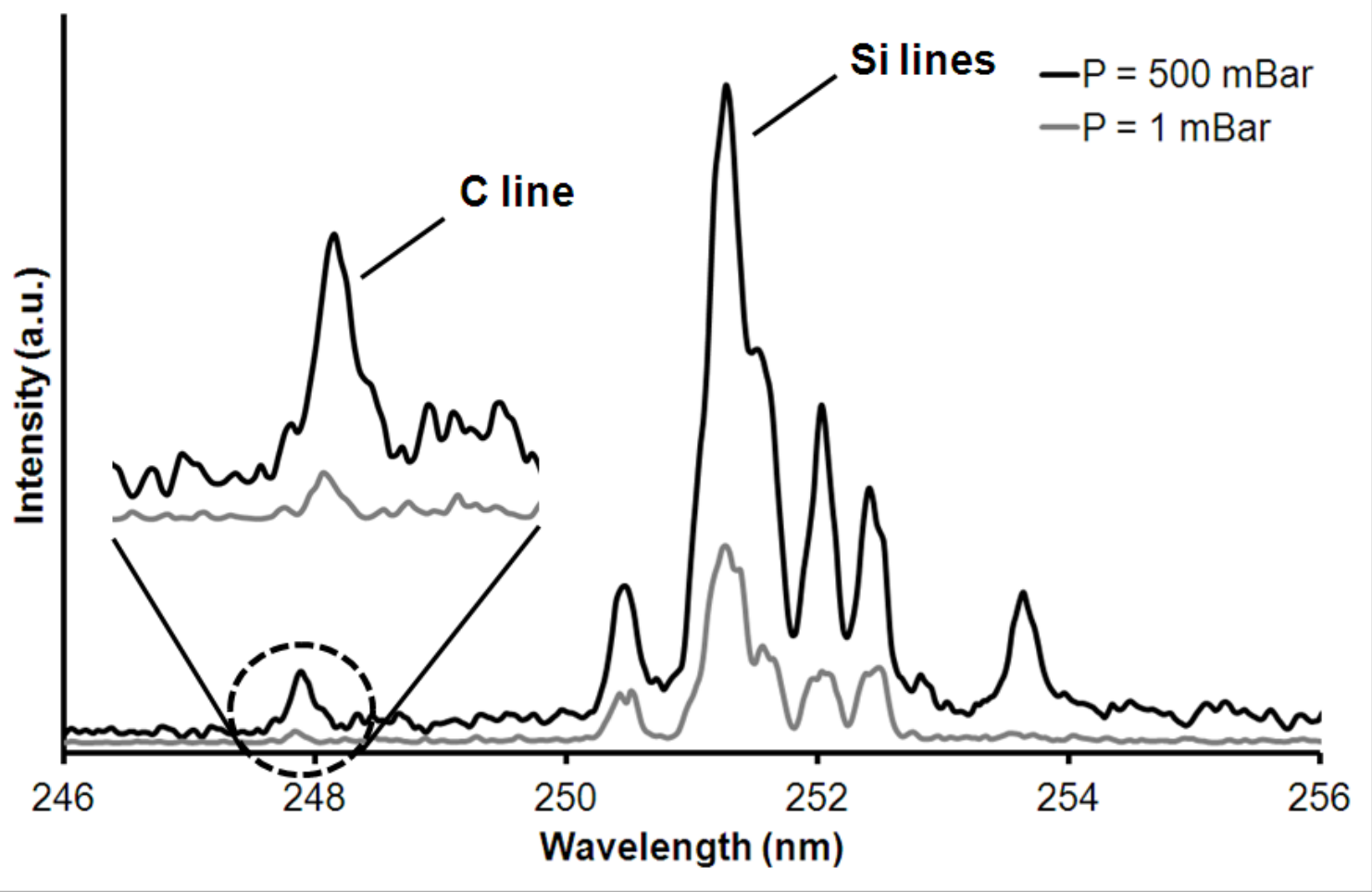

Figure 5 


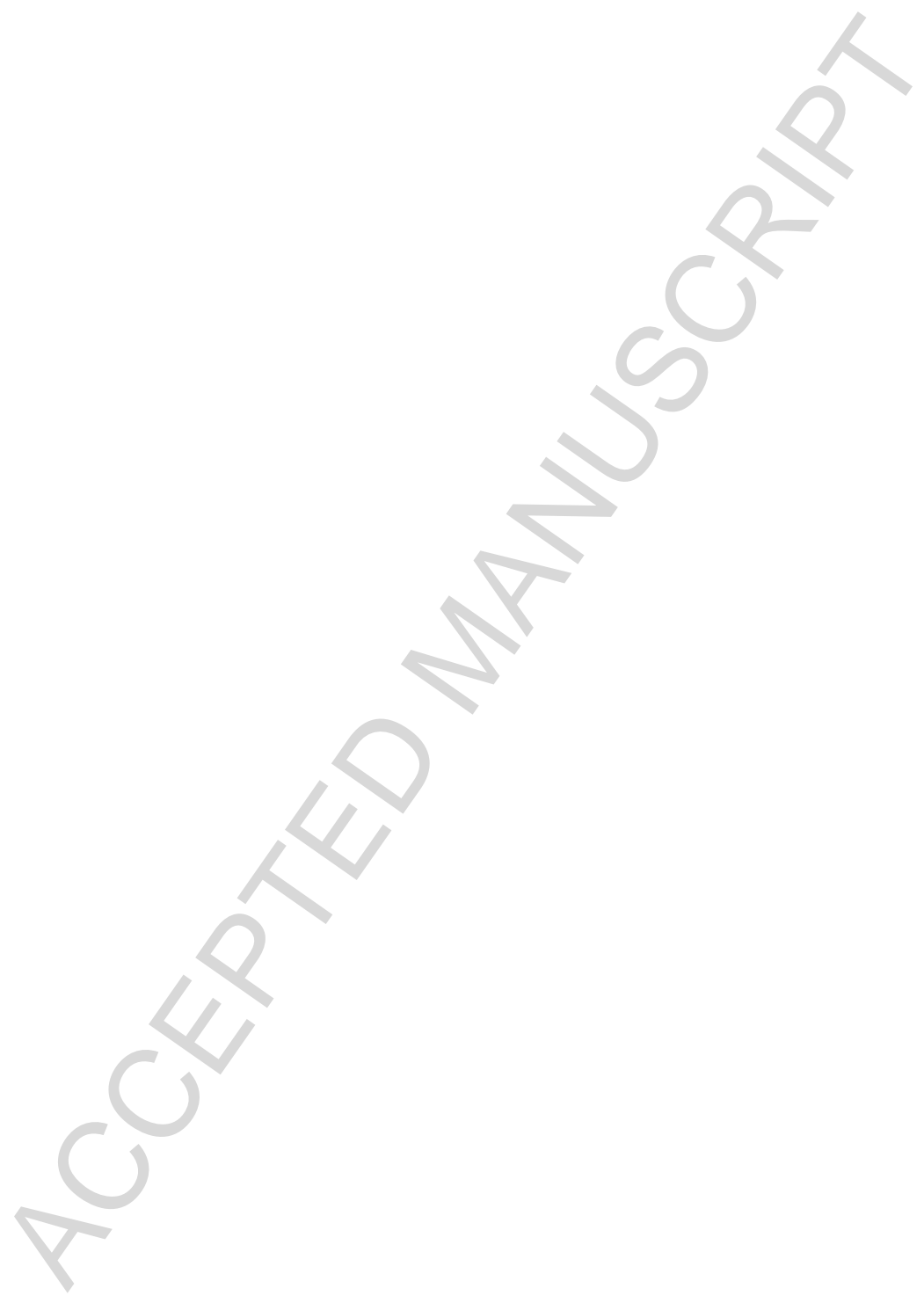




\section{HIGHLIGHTS}

- Determination of the elemental composition of composite nanoparticles combining a pulsed laser spectroscopic technique with a low pressure RF (Radio Frequency) plasma discharge.

- The nanoparticles are injected in the RF plasma discharge where they are trapped and maintained in suspension

- Determination of the elemental composition of the trapped particles is achieved through optical emission spectroscopy following irradiation by nanosecond laser pulses

- The analysis is done at reduced pressure, which is that of the RF discharge, namely $0.25 \mathrm{mBar}$. 